\title{
Soft Cells for Programmable Self-Assembly of Robotic Modules
}

\author{
Jürg Germann, Andrea Maesani, Ramon Pericet-Camara, Dario Floreano \\ Laboratory of Intelligent Systems, École Polytechnique Fédérale de Lausanne (EPFL), Station 11, \\ CH-1015 Lausanne, Switzerland
}

Keywords: Modular robotics, multi-cellular robotics, soft robotics, self-assembly, programmable selfassembly

\begin{abstract}
:
Programmable self-assembly of chained robotic systems holds potential for the automatic construction of complex robots from a minimal set of building blocks. However, current robotic platforms are limited to modules of uniform rigidity, which results in a limited range of obtainable morphologies and thus functionalities of the system. To address these challenges, we investigate in this paper the role of softness in a programmed self-assembling chain system. We rely on a model system consisting of "soft cells" as modules that can obtain different mechanical softness presettings. Starting from a linear chain configuration, the system self-folds into a target morphology based on the intercellular interactions. We systematically investigate the influence of mechanical softness of the individual cells on the self-assembly process. Also, we test the hypothesis that a mixed distribution of cells of different softness enhances the diversity of achievable morphologies at a given resolution compared to systems with modules of uniform rigidity. Finally, we illustrate the potential of our system by the programmable self-assembly of complex and curvilinear morphologies that state-ofthe-art systems can only achieve by significantly increasing their number of modules.
\end{abstract}




\section{Introduction}

Programmable self-assembly of chained robotic components is a promising strategy for the automatic construction of complex robots [1]. Inspired by protein folding, it comprises the self-assembly of morphologically and functionally diverse structures from a minimal set of building blocks. The major benefits of this universal method are: first, it is superior to other self-assembly strategies in terms of yield and the range of achievable morphologies [2,3]. Second, components can be relatively simple because there is a permanent connectivity constraint between all components, which reduces hardware needs (e.g. for communication or attachment/detachment) [4]. Third, because of its intrinsic modularity, it is especially suited for in silico optimization of morphology and functionality using stochastic meta-heuristics such as evolutionary algorithms [5]. Thus, such a method could lead to automatically designed robots that "walk out of the printer" [6].

Programmable self-assembly of chained robotic systems has been investigated by Griffith et al. [7], who suggested a two-dimensional system composed of serially connected square tiles. The tiles could move around their connection points and eventually fold into different geometric configurations. Four tiles having different magnetic patterns enabled the approximation of any two-dimensional shape. This work has been further expanded to three dimensions, along with algorithms to approximate any three-dimensional shape [8]. Inspired by that work, Knaian et al. [9] developed a system called "MilliMoteins" that consists of a chain of electropermanent magnetic motors that allows a chain to dynamically fold into different configurations. In another work [10], it was shown that serially connected soft cubes can self-assemble into a previously designed 3-D shape by simply being stretched. Finally, Risi et al. [11] presented software results of a custom "printer" that folds a long ribbon of material bearing additional elements such as motors and sensors. The authors evolved and optimized robots having different morphologies and performing different forms of locomotion.

All these systems demonstrate some potential of programmable self-assembly of robots, but yielding significant limitations: first, the obtainable morphologies of these systems are very limited, and second, their mechanical flexibility is not optimized. This is likely due to the fact that all the components have a predefined shape and uniform hardness.

Recently, a new emerging trend in robotics aims at stressing the importance of morphology and materials. "Soft robotics" focuses on the importance of intelligent mechanical design by taking inspiration from the many soft-bodied animals in nature that exploit the power of "mechanical intelligence". Soft robotics promises to produce simple and efficient robots for a variety of tasks because parts of the control computation can be outsourced to the mechanics of the system [12-14]. Applying the concepts raised in soft robotics to programmable self-assembly systems will likely overcome some limitations arising from constrained mechanical flexibility that yields restricted morphologies, and therefore, limited functionality.

In this paper, we systematically investigate the role of mechanical softness in a system that programmatically self-assembles. We introduce a model system that is unique because it is composed of "soft cells" that can obtain different mechanical softness presettings. With this system, our goal is to test the hypothesis that a mixed distribution of cells of different softness settings enhances the diversity of achievable morphologies at a given resolution compared to systems with uniform soft or hard components.

In the following, we first describe our system along with its most defining parameters. Subsequently, we characterize the self-assembly of basic clusters, i.e. the interaction between two and three cells, respectively. Finally, we show examples of programmable self-assembly using no more than sixteen cells that yield complex and curvilinear morphologies. Our results have been derived from simulated and macroscale hardware soft cells. 


\section{System Description}

\section{Design}

Our system consists of a chain of modules or "cells" that are permanently linked by free rotating joints (Figure 1a, left). A cell sequence is defined by the intrinsic properties of every individual cell. When released from an initial linear configuration, the chain self-folds as a result of the intercellular interactions. The final morphology of the system will only depend on the predetermined cell sequence (Figure 1a, right).

Every cell of the chain is composed of a uniformly thin membrane and has a predefined softness. A cell features specific connection areas around the membrane that allow a cell to interact with other cells. The arrangement of these areas on the cell surface is inspired by the work of Griffith $[7,8]$ which claims that a set of four different cell types with four connection areas per cell is enough to produce a large variety of morphologies. This connection pattern is reproduced in our model system using small magnets (Figure 1b).

In the chain configuration, cells fold at each of the permanent joints to the direction where their connection areas are attracting (Figure 1c, middle and right). As a consequence of the magnetic forces and the inherent cell softness, a certain contact area between two folded neighbor cells is formed. This resulting interaction with the subsequent formation of the intercellular contact area represents the basic folding process of our model system (Figure 1c, middle and right). Indeed, the combination of all basic folding processes in a whole chain results in a specific $2 \mathrm{D}$ structure.

We hypothesize that programming the softness of the individual cells can lead to morphologically more diverse structures and to an enhancement of the solution space of foldable shapes when compared to systems of uniform rigidity. This reasoning becomes more apparent when the selffolding of three cells is considered. Figure 1d displays the obtained angle $\alpha$ between cell centers for different cell softness settings. Despite the fact that both cell sequences have the same magnetic pattern, the obtained angle $\alpha$ is smaller when having softer cells (Figure 1d, right) with respect to harder cells (Figure 1d, middle). Thus, the obtained angle at every cell sequence can be controlled by tuning the softness of every individual cell.

\section{Implementation}

Software: We make use of a previously developed 2D simulator to aid in the design and control of a system based on entirely soft cells $[15,16]$. In this simulator, the membrane of soft cells is composed of multiple rigid bodies (see Figure 2a), aligned and connected by hinge joints to form a closed chain (Figure $2 b$ ). The hinges enable full flexibility of the membrane. We did not implement elasticity between the membrane body joints because this would easily result in cell inter-penetrations when bringing multiple soft cells together. The cell parametric softness is implemented in the simulations following a simple yet effective model based on thermodynamics [17]. In this model, we consider that the soft cell is filled with an ideal gas. The established internal pressure determines the softness of the cell (see Supplementary Information, section 1.1). In order to implement the interaction between adjacent cells, we enable a magnetic field generated by every membrane rigid body and characterized by a magnitude of the magnetic pole and an interaction radius. Thus, the membrane rigid body acts as a connector between neighboring cells. If two or more magnetic fields overlap, the connectors will attract or repel each other through a force-distance relation [18] (see Supp. Info, section 1.2).

Hardware: To further validate our concept in the real world, we developed a macroscale instantiation of the soft cells in hardware. This experimental system allows control over the resulting cell softness and an easy integration of connection points in predetermined arrangements. To produce the soft cells, we build rings out of Ecoflex 0030-A (Smooth-On Inc.) with an outer diameter of $5.5 \mathrm{~cm}$ and an inner diameter of $4.8 \mathrm{~cm}$ (Figure 3). The ring shape and size allows integrating smaller passive rings in the inner face of the cell to preset the softness in a simple way. The passive rings are fabricated in different thicknesses out of PDMS Sylgard 184 (Dow Corning, Inc.) (see Supplementary Figure S3). We rely on small cylindrical Neodymium permanent magnets for the membrane connectors (Webcraft $\mathrm{GmbH}$ ). The utilized magnets have a size of $2 \times 2 \mathrm{~mm}$ and a holding strength in contact of $150 \mathrm{~g}$. These dimensions were implemented as derived from results in simulation (see also Results and Supp. Info, section 2.1). 
Integrating multiple magnets into a supple material is difficult because magnets generally do not bond well to elastomers and the intersecting magnetic fields can cause the magnets to misalign. To sidestep this problem, we cast the magnets into PDMS using a cylindrical shape. Then, we glue the cylindrical pieces using a thin layer of PDMS into the perforated Ecoflex rings. The relatively high lateral blocking forces prevent the cylinders with the magnets from misaligning.

To connect multiple cells together as a permanently locked chain, we use small laser-cut acrylic pieces. These pieces are squeezed into the cells but not bonded, which is advantageous to reconfigure sequences fast and easily.

In order to have low friction during self-folding experiments, we glue small pieces of Velcro underneath the cells to reduce ground contact area (Figure 3b). A chemical bench serves as lowfriction ground.

\section{Results}

\section{Characterization of single fold between two cells}

The overall self-folding of the cell chain can be dissected into single folding processes happening between pairs of cells. Thus, investigating a single fold between two cells provides a basis to estimate the folding of longer chains. Our strategy consisted of realizing a full characterization of the system in simulation, assessing the effect of the different parameters on the single folding behavior. In addition, we realized an analogous characterization of the fabricated hardware in order to validate the overall interplay between single fold and cell softness in the case of a real world system.

We characterize the interaction between two folding soft cells via the effective contact area after folding (see Figure 4a). We study the effect of the cell parameters, namely, the mechanical softness and the magnitude of the magnetic pole of the membrane connection areas. Figure $4 \mathrm{~b}$ displays the obtained simulation results of the effective contact area between two cells (see Figure 1c and 4a) as a function of their mechanical softness and the magnetic force between two connectors in close contact. It is observed that the contact area between two cells increases with the softness of the cells, except for the case where the magnetic force is minimal. Also, an increase of the magnetic force results in an increase of the contact area at constant mechanical softness. The maximal contact area, i.e. when neighboring cells are in contact with a full quarter of their circumference, is reached for a variety of settings when one or both of the cells are sufficiently soft and the magnetic force is strong enough. The lowest value for the contact area (approximately 0.2 of a quarter cell circumference) is obtained when both cells are at maximum hardness and the magnetic force is minimal.

An important outcome of this experiment is the condition in terms of magnetic force at which the influence of the cell softness is maximized after the folding process. Indeed, one of the goals of this work is to achieve a modular system where the space of attainable morphologies with a reduced number of cells is enhanced thanks to the tunability of the cell softness. The simulation data yield that the widest range of different values of the contact area at a predetermined magnetic force, that is, where the effect of cell softness is maximized, is obtained when the magnetic force is at $1.5 \mathrm{~N}$. The simulation results of the effect of cell softness on contact area for this specific magnetic value are displayed in Figure 4c.

Furthermore, we investigate the influence of cell softness on the experimental contact area between the hardware cells at five different softness values (Figure 4d). For this, we implement hardware magnets producing the magnetic force that yields a maximum range of contact areas as found in simulations, that is, $1.5 \mathrm{~N}$ (see Supp. Info, section 4). In this, it is observed that the cell contact area increases with the softness of the cells, as found in simulations (Figure 4c). However, a lower value of contact area is obtained in hardware cells, i.e. 0.35 of a quarter cell circumference in hardware vs. 0.55 in software. This is likely due to differences in the absolute maximum hardness of the simulated and hardware cells. We must also bear in mind the different origin of their elastic properties (see Supp. Info, section 1.1 and 2.1) reflected in a slightly dissimilar elastic behavior (see Figure S1).

\section{Exploiting cell softness to self-assemble curvilinear morphologies}

We present here the programmable self-assembly of long chains of cells into a variety of preset 
morphologies. This experiment illustrates the capabilities of our system to achieve such morphologies with a reduced number of cells if their softness can be tuned.

We designed four linear sequences of non-uniformly soft cells to fold into the letters "E","P","F", and "L", replicating the design of the EPFL logo (Figure 5a). The cell sequences for each target shape are obtained by extracting the sequence of necessary single folds to produce it. Then, the cell parameters that yield every single fold are estimated from the characterization results (Figure $4 \mathrm{c}$ and $\mathrm{d}$ ) and manually encoded in the cell sequence.

Figure $5 \mathrm{~b}$ and $5 \mathrm{c}$ show pictures of different self-folding time steps of the four exemplary chains in simulation and hardware, respectively. In both hardware and software experiments, we recognize similar folding dynamics and final configurations. This is achieved despite the different cell elastic behavior, the presence of ground friction in hardware, and the increased mass in stiffer hardware cells. In these experiments, the system self-folds into a wide range of challenging morphologies with optimal cell resolution. For instance, the folding of the letter "E" demonstrates the formation of symmetric forms, filled entities, such as the six cells in the middle of the sequence, and variable angles between three cells. The folding of the letter "P" illustrates how round shapes can be obtained with a small set of cells. Further, the folding of the letters "F" and "L" show again the folding of curvatures with arbitrary angles, asymmetric forms, and filled forms.

\section{Discussion}

Our experiments demonstrate that the interaction between folding cells, and more specifically, the contact area, can be mediated to a high extent by presetting non-uniform softness values at every individual cell. This is essentially different from state-of-the-art systems that, due to uniform softness of the cells, always fold with constant contact area. This factor limits the range of attainable morphologies for low module numbers.

The gain and the advantage of our system are illustrated by examples of folded curvilinear morphologies at low resolution. Various shape features like curvatures with almost arbitrary angles, round circles, straight lines, sharp corners, continuous filled areas as well as shapes with holes are all enabled with a very low number of cells. We interpret this as an increase of morphological diversity at a given resolution with respect to the state of the art. To further support the advantageous properties of our system, we have computed the theoretical maximum shape complexity, which is defined by the entropy of curvature [19] (see Supp. Info, section 3), that can be achieved for a given number of parts bearing uniform and non-uniform softness distribution. For the latter, we assume that the number of possible softness states of a cell is always larger than the number of available parts. In the results shown in Figure 6, it is observed that the theoretical maximum shape complexity of non-uniform softness chains increases logarithmically, thus yielding a fast increase for a low number of parts. On the other hand, the complexity of configurations bearing uniform softness distribution saturates already at chains of five parts. Therefore, we can surely claim that a non-uniform softness distribution of the robotic parts of our system enables the self-assembly of more complex morphologies at lower production costs -because less modules are required-, at shorter folding times -because the number of folds is also lower-, and potentially, at lower energetic cost -due to the two previous reasons combined-. In Figure 6, the shape complexity values for the chain configurations of the EPFL letters have also been plotted for evaluation. In particular, it is observed that the shape complexity of the letter " $\mathrm{E}$ " is achievable only if the system bears a non-uniform softness distribution.

In this work, the specific sequences of cells of the provided folding examples were found manually. In order to exploit the newly available design space of foldable morphologies, it will be important to develop methods to automate the step of finding the optimal sequence that yields the target morphology.

For hardware implementations, an important setback to overcome is the presence of non-linear interactions. In our example experiments, we let a chain of cells fold in parallel, i.e. the folds occur simultaneously. This obviously leads to additional, often non-linear interactions between the folding cells, which may complicate the prediction of the outcome. A potential solution is to force the folds to occur sequentially, e.g. by introducing a "printer"-like system as suggested in [7]. 
Additionally, an important consideration to explore is the scalability of the concept presented here, for instance, by extending our system to 3D. Considering that the essential principles of the concept would remain, e.g., the interplay between cell softness and contact area, the solution space of morphologies, and with this, the system complexity, will be dramatically enhanced by adding a third dimension. This is due to the fact that, in this case, the folding events could happen in the three directions of space.

Finally, programmable self-assembly is an interesting technique for building complex micro- and nano-structures [20,21]. Indeed, micro- and nanoscale components acting as modules can be produced with a number of state-of-the-art techniques (e.g. inkjet printing [22], MEMS fabrication [23], etc.). Materials and methods to vary the softness of micro-particles do exist [24,25], which implies at least a potential scalability of our concept to microscopic dimensions.

\section{Conclusion}

In this paper, we investigated the effect of module softness in the programmable self-assembly of robotic chains. So far, research on programmable self-assembly has been limited to the use of cells with uniform rigidity. In our system, we alleviate this constrain by enabling the programming of the softness of every cell. We show that the cell softness can mediate the local interactions between cells, and with this, the folded morphology of a complete chain. Finally, we demonstrate that a non-uniform softness distribution among the cells enhances the diversity and complexity of the achievable morphologies of complete folded chains. Thus, this opens the door to a wider design space for programmable self-assembly.

The increase in morphological diversity of foldable robotic chains at a given resolution may permit the accomplishment of enhanced functionality, lower fabrication complexity and costs, and a more efficient use of components. However, new automatized methods will be needed to optimize the extraction of the required cell sequence to achieve increasingly complex morphologies. To finish, we envision a future integration of sensors, actuators and other electronic components into the soft cells of the presented system that would allow the automatic design and construction of complex soft robots by programmable self-folding.

\section{Acknowledgements}

We would like to thank Stefano Mintehev, Stefano Varricchio, Bryan Schubert and Pavan Ramdya for proofreading the manuscript and giving constructive feedback. Furthermore, we acknowledge the contributions of Manuel Stöckli on the software development and Jonathan Zuercher on the hardware implementation. This research is supported by the Swiss National Foundation through the National Center of Competence in Research Robotics (NCCR).

\section{Author Disclosure Statement}

No competing financial interests exist.

\section{References}

[1] Murata S, Kurokawa H. Self-Organizing Robots. Springer Tracts in Advanced Robotics. 2012; 77

[2] Whitesides G M, Grzybowski B. Self-assembly at all scales. Science 2002;295:2418-2421

[3] Pelesko J A. Self assembly: the science of things that put themselves together. CRC Press, 2007

[4] Gross R, Dorigo M. Self-Assembly at the Macroscopic Scale. Proceedings of the IEEE 2008;96: 1490-1508

[5] Pfeifer R, Lungarella M, Iida F. Self-Organization, Embodiment, and Biologically Inspired Robotics. Science. 2007;318:1088-1093

[6] Lipson H, Pollack J B. Automatic design and manufacture of robotic lifeforms. Nature 2000;406: 974-978

[7] Griffith S. Growing Machines. PhD Thesis, MIT, 2004 
[8] Cheung K C, Demaine E D, Bachrach J R, Griffith S. Programmable assembly with universally foldable strings (Moteins). IEEE Transactions on Robotics. 2011;27:718-729

[9] Knaian A N, Cheung K C, Lobovsky M B, Oines A J, Schmidt-Neilsen P, Gershenfeld N A. The milli-motein: A self-folding chain of programmable matter with a one centimeter module pitch. IEEE International Conference on Intelligent Robots and Systems. 2012;1447-1453

[10] Yim S, Sitti M. SoftCubes: Towards a Soft Modular Matter. IEEE International Conference on Robotics and Automation. 2013;530-536

[11] Risi S, Cellucci D, Lipson H. Ribosomal Robots: Evolved Designs Inspired by Protein Folding. GECCO '13. 2013

[12] Trivedi D, Rahn C D, Kier W M, Walker I D. Soft robotics: Biological inspiration, state of the art, and future research. Applied Bionics and Biomechanics. 2008;5:99-117

[13] Kim S, Laschi C, Trimmer B. Soft robotics: a bioinspired evolution in robotics. Trends in biotechnology. 2013

[14] Lipson H. Challenges and Opportunities for Design, Simulation, and Fabrication of Soft Robots. Soft Robotics. 2013;1:21-27

[15] Germann J, Maesani A, Stöckli M, Floreano D. Soft Cell Simulator: A tool to study Soft MultiCellular Robots. IEEE International Conference on Robotics and Biomimetics, 2013; 1300-1305

[16] Catto E. Box2d: A 2d physics engine for games available at www.box2d.org

[17] Matyka M, Ollila M. Pressure model of soft body simulation. Proceedings of Sigrad. 2003; 29-34

[18] Resnick R, Halliday D, Walker J. Fundamentals of physics. John Wiley. 2008

[19]| Page D L, Koschan A F, Sukumar S R, Roui-Abidi B, Abidi M A. Shape analysis algorithm based on information theory. IEEE International Conference on Image Processing, 2003; 229-232

[20] Qi H, Ghodousi M, Du Y, Grun C, Bae H, Yin P, Khademhosseini A. DNA-directed selfassembly of shape-controlled hydrogels. Nature communications, 2013; 4

[21] Deschner R, Tang H, Allen P, Hall C, Hlis R, Ellington A, Willson C.G. Polyfunctional Microscale Particles for Programmed Self Assembly. Chemistry of Materials, 2014

[22] Singh M, Haverinen H M, Dhagat P, Jabbour G E. Inkjet printing-process and its applications. Advanced materials, 2010; 22(6), 673-685

[23] Gad-el-Hak, M. The MEMS handbook. CRC press, 2010

[24] Sunyer R, Jin A J, Nossal R, Sackett D L. Fabrication of hydrogels with steep stiffness gradients for studying cell mechanical response. PloS one. 2012;7:10:e46107

[25] Sant S, Hancock M J, Donnelly J P, Iyer D, Khademhosseini A. Biomimetic gradient hydrogels for tissue engineering. The Canadian journal of chemical engineering, 2010; 88(6), 899-911

Address correspondence to:

Prof.Dario Floreano

Laboratory of Intelligent Systems

LIS - IMT - STI - EPFL

ELE 138, Station 11

1015 Lausanne, Switzerland

E-mail: dario.floreano@epfl.ch 
a

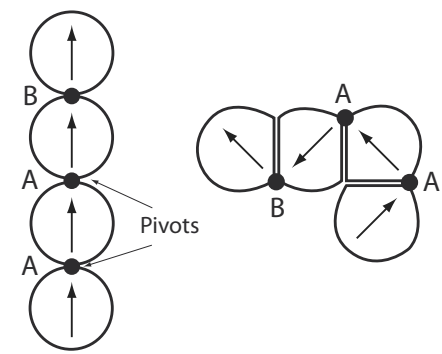

b

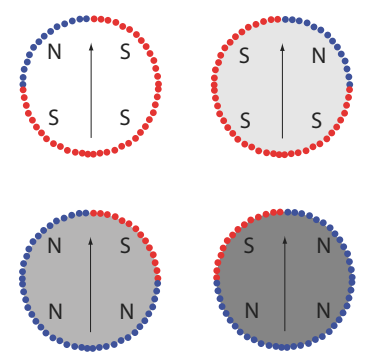

c
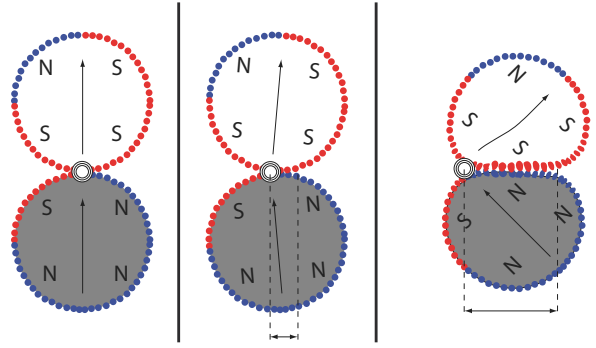

d

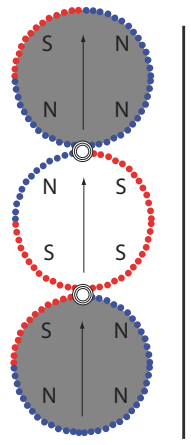

Initial

configuration

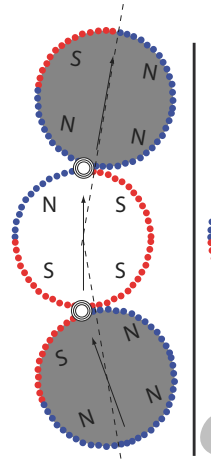

Hard cells

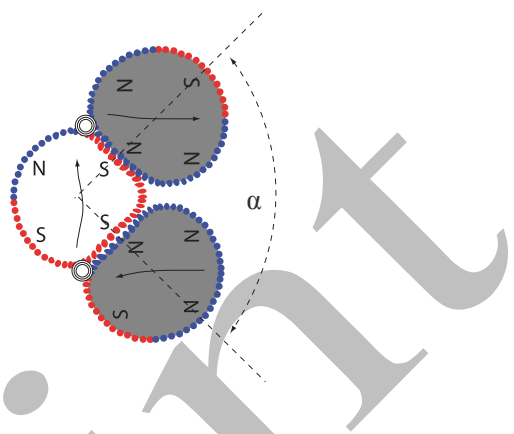

Soft cells

Figure 1: (a) Basic working principle of programmable self-assembly of linear chains: modules are aligned and connected through permanent pivot joints. Depending on local interactions, the chain folds at the pivot points. The output of the selffolding example is shown on the right. (b) Design of soft cells: the cells have four magnetic sections $(\mathrm{N}$ and $\mathrm{S}$ for magnetic north and south pole, respectively). According to the distribution of the magnetic regions, four different cell types have been designed. The black arrow indicates the orientation of the cells when connecting multiple cells together; (c) Schematic representation of folding adjacent soft cells at initial configuration (left), final configuration for two hard cells (middle), and for two soft cells (right); (d) angle $\alpha$ between three adjacent cells at initial configuration (left), final configuration for three hard cells (middle), and for three soft cells (right).

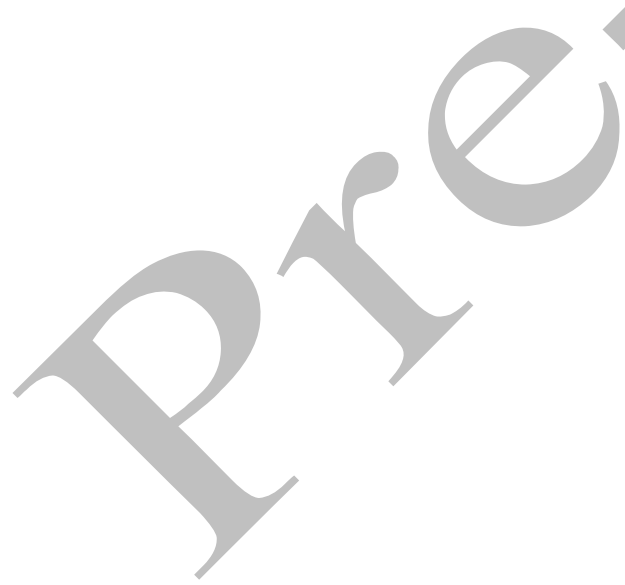




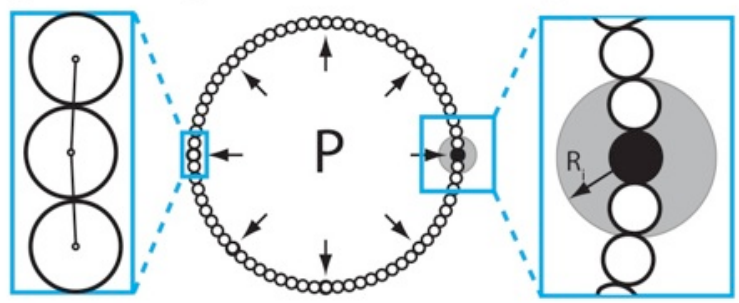

Figure 2: Soft cell modeling: (a) cells are composed of multiple small rigid bodies (black circles) and have parametric softness based on an internal pressure $\mathrm{P}$ acting on the rigid bodies, (b) the rigid bodies of the membrane are connected by hinges, (c) each membrane body may have a connection mechanism (black filled circle) with a specific interaction radius $R_{i}$ around the rigid body (grey circle).
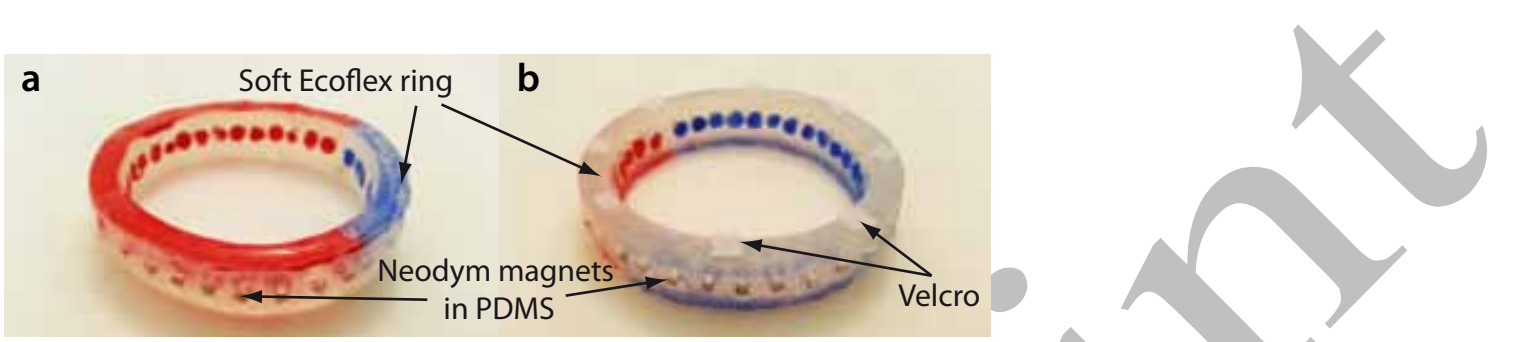

Figure 3: (a,b) Fabricated soft cells showing the Neodym magnets integrated in the cell membrane. (b) Small pieces of Velcro are glued underneath the cell to reduce ground friction.

a

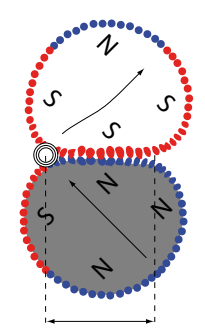

C

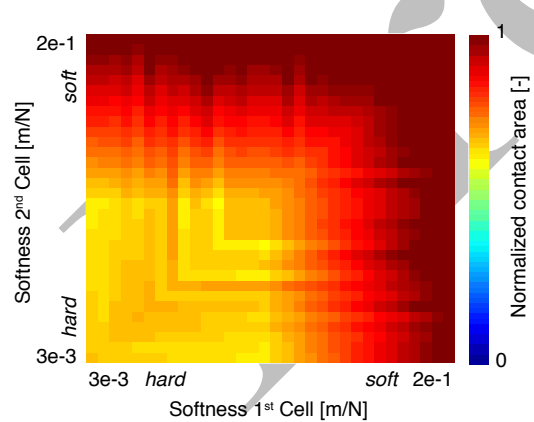

b

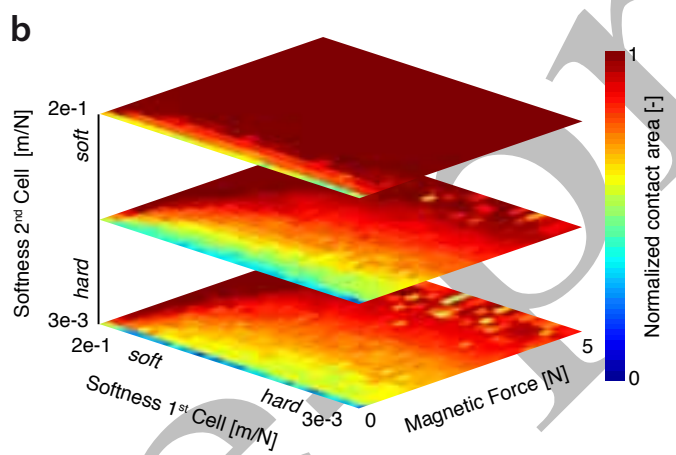

d

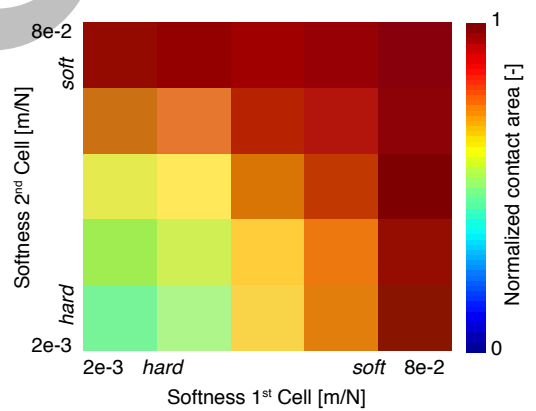

Figure 4: (a) Scheme of two neighboring cells after folding showing the defined contact area. (b) Contact area in equilibrium between two neighboring cells (color scale) as a function of the softness of each cell and of the magnetic force as measured in simulations. (c) Contact area in equilibrium between two neighboring cells (color scale) as a function of the softness of each cell for a magnetic force of $1.5 \mathrm{~N}$ as measured in simulation. (d) Contact area in equilibrium between two neighboring cells (color scale) as a function of the softness of each cell as measured with the hardware prototype. 
a
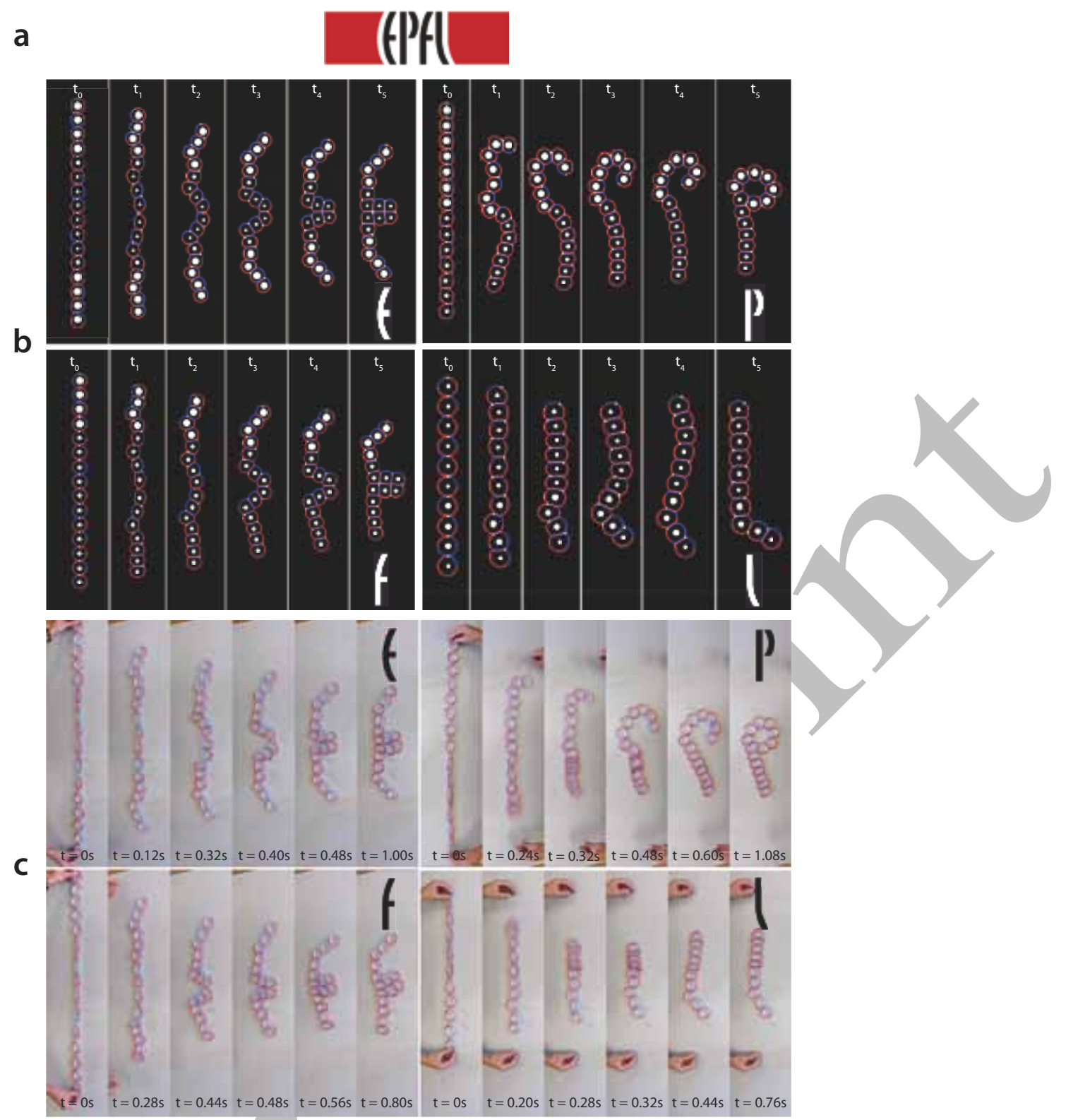

Figure 5: Demonstration of self-folding curvilinear morphologies. (a) Original EPFL-logo featuring round letters. (b) Simulation of the self-folding of four chains to form the letters "E" (top left), "P" (top right), "F" (bottom left), and "L" (bottom right) of the EPFL logo. The white dots in the figure indicate the softness of the cells, where bigger dots corresponds to lower softness. The letters $t_{0}-t_{5}$ indicate the sequence time. (c) Experimental self-folding using the same the programmed sequence in simulation. 


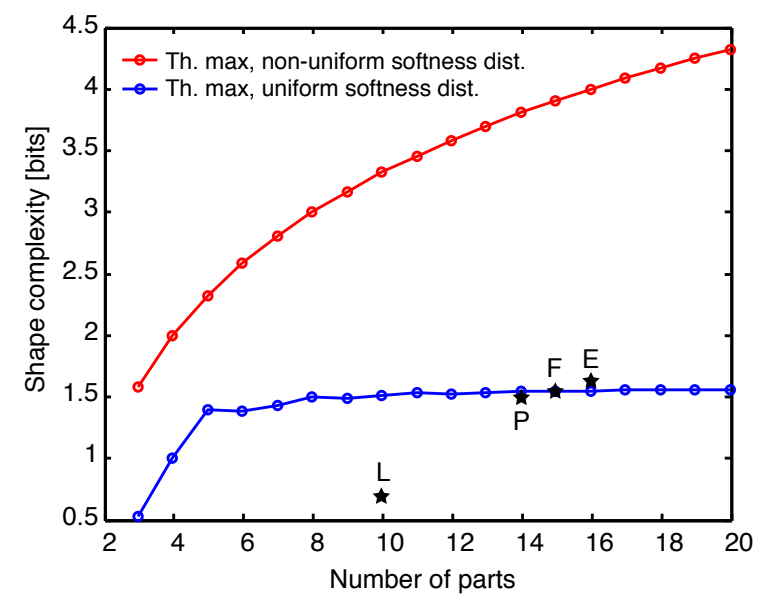

Figure 6: Shape complexity of soft self-folding chains as a function of the number of parts. Connected circles display the theoretical maximum complexity achievable for non-uniform (in red) and uniform (in blue) cell softness distributions. The stars indicate the shape complexity of the self-folded "EPFL" letter sequences. 


\section{Supplementary Information}

\section{Simulation details}

We developed a 2D simulator to aid in the design and control of a system based on entirely soft cells [15]. The requirements on the simulator were (i) high enough simulation speed to test systems with a large number of cells, (ii) large non-linear deformations without module self-penetration, (iii) tunability of cell softness, and (iv) physics-based active module connectivity (e.g. magnetic connectivity).

Our implementation is based on the Box2D physics engine [16], which is an open source rigid body solver written in $\mathrm{C}++$. Box2D is stable, adaptable, computationally efficient, and has an already builtin visualization testbed. Using Box2D we developed the Soft Cell Simulator (SCS) by implementing a soft cell modeling and enabling inter-module connectivity. SCS is a completely object-oriented environment, thus allowing easy integration of different types of connectors or soft module modelings. ${ }^{1}$

\subsection{Pressure based model for soft cells}

The membrane of soft cells is composed of multiple rigid bodies (Figure 1a). These bodies are aligned and connected by hinge joints to form a closed chain (Figure 1b). The hinges enable full flexibility between the membrane bodies. Deliberately, we did not enable elasticity between the small membrane bodies, because this would easily result in cell inter-penetrations when trying to bring multiple soft cells together. The cell parametric softness is implemented in the simulations following a simple yet effective model based on thermodynamics [17]. In this model, we consider that the soft cell is filled with a gas. The established internal pressure determines the softness of the cell.

In principle, pressure is the force acting on incremental surface elements and is normal to the surface. The expression for pressure in a specified point on a surface is then:

$$
\vec{P}=P \vec{N} \quad \text { (Equation 1) }
$$

where $\mathrm{P}$ is a pressure value and $\mathrm{N}$ is the normal vector to the surface which the pressure force is acting. In order to obtain the pressure force, we multiply the pressure value with the area of the surface A. This leads to the expression:

$$
d \vec{F}=\vec{P} d A \quad \text { (Equation 2) }
$$

To calculate the pressure force value $\mathrm{P}$, we use the thermodynamic approximation known as the Ideal Gas Law. This approximation focuses more on the macroscopic effects of the gas activity, and does not take into account the actual interactions among gas particles. The Ideal Gas Law gives a simple relation between pressure $P$, gas temperature $T$, body volume $V$, amount of substance of gas $n$ and the ideal gas constant $R$ :

$$
P=\frac{n \cdot R \cdot T}{V}
$$

(Equation 3)

We assume temperature to be constant and only the volume of the soft body changes. Thus, for every simulation step we compute the variation of the soft body volume and apply a reactive force due to internal pressure on every membrane body

$$
F_{\text {react }}=C \cdot\left(\frac{V_{\text {init }}}{V}-1\right)
$$

where $\mathrm{C}$ is the constant defining the internal pressure, $\mathrm{V}_{\text {init }}$ the initial volume and $\mathrm{V}$ the actual volume of the soft body.

For all simulations in this article, we use soft cells with membranes composed of a hundred rigid bodies, which is a compromise between simulation speed and accuracy.

We test the implementation of the simulation and characterize the influence of the pressure constant $\mathrm{C}$ by performing a force-deflection measurement as illustrated in Supplementary Figure S1a. In this test,

\footnotetext{
${ }^{1}$ All source code is freely available at: https://github.com/lis-epfl/scs
} 
the deflection for cells of different softness is measured under gradually increasing loads. The obtained results for 12 different pressure constants C are shown in Supplementary Figure S1b. As can be seen in the graph, the relation between force and deflection resembles the one of a progressive spring, which is reasonable given the modeling based on the pressure of an ideal gas. Also, it is observed that the deflection of the cell decreases considerably with an increase of the internal pressure. We extract a softness value for each different pressure constant $\mathrm{C}$ settings by linearizing the curves in Supplementary Figure S1b for the plotted range. The results of this linearization for five different settings - ranging from maximum softness to maximum stiffness - are shown in Supplementary Table S1.

\subsection{Connection mechanism}

In order to implement the interaction between adjacent cells, we enable a magnetic field generated by every membrane rigid body and characterized by the magnitude of the magnetic pole and an interaction radius. In this interaction radius, a magnetic field around the connector applies. If two or more connector magnetic fields overlap, they attract through a force-distance relation [18]:

$$
F=\frac{\mu q_{m 1} q_{m 2}}{4 \pi r^{2}}
$$

where $\mu$ is the permeability of the intervening medium (here: air), $q_{m 1}$ and $q_{m 2}$ are the magnitudes of magnetic poles and $r$ is the separation of the magnets. For the self-assembly experiments, there is one connection mechanism fixed to every small rigid body of a cell membrane.

In Figures 4 and S3, where the influence of the magnetic pole magnitude is displayed, we plot the magnetic force when two connectors are in close contact (i.e. when $r$ is equal to the diameter of a small rigid body of the membrane).

\subsection{Simulation procedure}

For the experiments in simulation, we pre-align cells in a chain before applying any forces to the system. The cells are sorted given the programmed sequence and connected through permanent joints. We assume a totally frictionless, gravity-free and unconstrained environment, and the only driving forces are the magnetic attractions between the cells and the elastic force between cells during contact. The folds between all the cells happen in parallel. For each experiment, we first run the simulation for 2000 simulation steps to let the folding complete and then perform the measurement for a duration of 200 simulations steps and average the results. The averaging is necessary even though the simulator is deterministic because we still notice small motions after completion of the folding process caused by the physics engine (numerical integration artifacts that arise with the use of a finite time step).

The ranges of magnetic attractions and cell softness implemented in our simulation experiments have been established such that the process does not fall in an unstable regime.

\section{Hardware details}

\subsection{Fabrication of soft cells}

To validate our concept in a real world environement, we developed a fabrication method to produce model macroscale soft cells. The main criteria for the fabrication method were to allow control over the resulting cell softness and to allow an easy integration of connection points in predetermined arrangements.

To obtain cells with controllable softness, we build rings out of Ecoflex 0030-A (Smooth-On Inc., Easton PA, US) with an outer diameter of $5.5 \mathrm{~cm}$ and an inner diameter of $4.8 \mathrm{~cm}$ (Supplementary Figure S2). The ring shape and size allows integrating smaller passive rings to change the cell softness in a simple way. The passive rings are fabricated in different thicknesses out of PDMS Sylgard 184 (Dow Corning, Midland MI, US) (see Supplementary Figure S2d). The softness of a cell can be mediated by dimensioning the size of the cylindrical rings. The ring stiffness can be calculated as [1]

$$
S_{\text {ring }}=\frac{E I}{D_{m}^{3}}
$$


where $E$ is the young's modulus of the ring material and $D_{m}$ is the mean diameter of the ring. $I$ is the second moment of area and is expressed for a cylinder as

$$
I=\frac{\pi}{4}\left(r_{o}^{4}-r_{i}^{4}\right) \quad \text { (Equation 7) }
$$

where $r_{o}$ is the outer radius and $r_{i}$ the inner radius of the ring. The force-deflection profile of a ring is then given as

$$
F=\frac{S_{\text {ring }} h}{d}
$$

where $F$ is the required force for a given deflection $d$ and $h$ is the height of the ring. The theoretical profile of the dimensioned rings is plotted in Supplementary Figure S1d.

We test the softness of the fabricated cells by performing a force-deflection measurement. The hardware setup is composed of a force sensor coupled to a linear stage. For the measurement, the cell is put in-between a constraining surface and the force sensor while the stage moves the sensor at a speed of $1000 \mathrm{um} / \mathrm{sec}$ (Supplementary S1c). The obtained data for the different softness settings and maximum normalized deflection of 0.5 is shown in Supplementary Figure S1d. As can be seen in the plot, the data is comparable to the analytical solution. The profile for the tested deflection regime is comparable to a linear spring and not a progressive spring as in simulation. This is mainly because the cells are empty inside and contain no gas (or mass) that would cause a more progressive behavior. Similarly as in simulation we extract a softness value for every softness setting. The five extracted values are compared to the ones found in simulation in Supplementary Table S1. Most noticeably, the maximum and minimum cell softness values are in a similar range.

We rely on small cylindrical Neodym permanent magnets for the membrane connectors (Webcraft $\mathrm{GmbH}$, Uster, Switzerland). Using permanent magnets means that the adhesion force is fixed. Therefore, this parameter has to be chosen carefully for successful self-folding. The number of magnets and their strength should maximize the contact area between two highly soft cells and minimize the contact area between two stiff cells. To simplify and speed up fabrication, we reduce the total number of connectors from 96 in simulation to 32 (Supplementary Fig. S2a-b). To cope with this reduction, the minimal connection strength should be increased because of the additional separation between connectors of the same cell. The magnets being used have a size of $2 \times 2 \mathrm{~mm}$ and a holding strength of $150 \mathrm{~g}$. This value is derived from results in simulation as described in the Results section. Integrating multiple magnets into a supple material is difficult because magnets generally do not bond well to elastomers and the intersecting magnetic fields can cause the magnet misalignment. To sidestep this problem, we mold the magnets into stiff PDMS using a cylindrical shape. Then, we glue the cylindrical pieces using a thin layer of PDMS into the perforated Ecoflex rings. Relatively high lateral blocking forces prevent the cylinders with the magnets from misaligning.

To connect multiple cells together as a permanently locked chain, we use small laser-cut acrylic pieces. These pieces are squeezed into the cells but not bonded, which brings the advantage to reconfigure sequences fast and easily.

In order to have low friction during self-folding experiments, we glue small pieces of Velcro underneath the cells to reduce ground contact area (Supplementary Fig. S2b). A chemical bench serves as low-friction ground.

\subsection{Experimental procedure}

For the hardware experiments, the cells are first connected as a chain with an established sequence of connection areas and softness parameters, and then stretched by hand such that no magnets are connected. Once released, the chain self-folds in the equilibrium state. We take pictures of these experiments and use common image processing software (Adobe Photoshop 4.0) to extract the contact area and folded angle along the cell sequence. For each different experimental setting we always perform 5 experiments and then compute mean and standard deviation of the measurement.

\section{Entropy of Curvature}


In order to assess the complexity of self-folded chains, we make us of Shannon's Entropy [2]. This has been already used to measure the complexity of objects $[3,4,5]$. Shannon's Entropy measures the amount of information of a random variable. To measure shape complexity, the curvature of the object is considered as a random variable. Remarkably, this measure strongly correlates with human notions of complexity $[4,5]$.

We present here briefly the main concept of the measure. Further details can be found elsewhere [3] [4]. Given a random variable $x$ with a probability density function (PDF) $p(x)$, entropy $H$ is defined as

$$
H=-\int_{-\infty}^{\infty} p(x) \log p(x) d x \quad \text { (Equation 9) }
$$

where $p(x)$ is a continuous function. If instead $p(x)$ is discretized such that $p_{i}=\int_{x_{i-1}}^{x_{i}} p(x) d x$ where $x_{\mathrm{i}}$ are specific values of $x$, then $H$ can be formulated discretely as

$$
H=-\sum_{i} p_{i} \log p_{i} \quad \text { (Equation 10) }
$$

Now, we consider discrete two-dimensional curves. Under the assumption that line segments are uniformly distributed across the curve, the curvature is directly proportional to the turning angle formed by the line segments. In order to estimate the PDF of the curvature function, a number of bins $\mathrm{M}$ are chosen and associated with a probability. The bin probability can be formulated as

$$
p_{i}=\frac{B_{i}}{N}
$$

(Equation 11)

where $B_{\mathrm{i}}$ is the number of samples that have the same curvature and $N$ is total number of samples. These definitions allow computing the entropy for various shapes.

In order to compute the shape complexity of self-folded cell chain, we assume that the number of parts is equal to the number of samples and that the curvature of a folded morphology is the line that connects the cell centers of adjacent cells.

To determine the theoretical maximum shape complexity for a given number of parts, we assume that the number of obtainable softness states of a cell for a non-uniform softness distribution is always larger than the number of available parts. Then, we obtain the maximal values when maximizing the PDF as given in Equation (10).

\section{Characterization of folding of three adjacent cells}

In further experiments, we also characterize the influence of a non-uniform softness distribution among the cells on the angle $\alpha$ between three adjacent cells (Supplementary Figure S3). This characterization supports our understanding of the essential functioning of folding soft cells and serves also as a basis to estimate the folding of longer chains.

Results are collected in simulation when varying the softness of the three cells and the magnetic pole magnitude of the connections. The simulation data allows again extracting an optimal magnetic force in contact that maximizes the influence of variable cell softness. The found magnetic force is very similar to the one found in the contact area experiments. Thus, that magnetic pole magnitude is implemented in our hardware model system .

Supplementary Figure S3b displays a 2D surface plot of the simulation results of the angle $\alpha$ after folding versus cell softness and the magnetic force between two connectors in close contact. The angle $\alpha$ decreases continuously with increasing softness for most of the measured magnetic forces. The highest angle of $148^{\circ}$ is found at minimum softness and maximum magnetic force, and the lowest angle of $87^{\circ}$ for a maximum softness at minimum magnetic force. The highest change of the angle $\alpha$ for the same magnetic force is attained when the force is about $1.5 \mathrm{~N}$. This value is similar to the optimal magnetic force to maximize the range of contact areas shown in the main body text $(1.5 \mathrm{~N})$.

We reproduce the experiments using our hardware. Supplementary Fig. S3c shows the mean and standard deviation of the angle $\alpha$ after folding versus the normalized softness of the cells. The obtained angle for different cell softness conditions ranges between $90^{\circ}$ to $140^{\circ}$, thus giving an 
overall range of $50^{\circ}$. This range could be further increased by stiffening the soft outermost part (Ecoflex layer) of the fabricated cells.

Supplementary Fig. S3c allows comparing results obtained in simulation and in hardware. Both dataset curves show a continuous decrease of the folded angle with an increase of the cell softness. The differences in the two curves is due to differences in elastic behaviors of the cells, deviations in the absolute stiffness of the cells and variations of the modeled and real magnetic forces.

The simulation and experimental results shown here support our claims that an increased morphological diversity (here expressed as the angle $\alpha$ ) can be obtained through a non-uniform softness distribution among the cells.

\section{References}

[1] Goodier J N, Stephen Prokofevich Timoshenko S P. Theory of elasticity. Engineer. 1878-1972

[2] C. E. Shannon C E. A mathematical theory of communication. Bell system technical journal. $1948 ; 27$

[3] Page D, Koschan A, Sukumar S, Roui-Abidi B, Abidi M. Shape analysis algorithm based on information theory. IEEE International Conference on Image Processing. 2003; 1; 229-232

[4] Sukumar S, Page D, Koschan A, Abidi M. Towards understanding what makes 3d objects appear simple or complex. IEEE International Conference on Computer Vision and Pattern Recognition. 2008

[5] Auerbach J E, Bongard J C. On the relationship between environmental and morphological complexity in evolved robots. International conference on Genetic and evolutionary computation conference. 2012; 521-528

Table S1: Extracted softness of software and hardware cells

\begin{tabular}{lll}
\hline Qualitative & Software & $\begin{array}{l}\text { Hardware } \\
\text { Cell Softness }\end{array}$ \\
Cell Softness $[\mathrm{m} / \mathrm{N}]$ & Cell Softness $[\mathrm{m} / \mathrm{N}]$ \\
\hline max soft & $1.6 \mathrm{e}-1$ & $8.3 \mathrm{e}-2$ \\
soft & $5.9 \mathrm{e}-2$ & $2.8 \mathrm{e}-2$ \\
mid & $2.2 \mathrm{e}-2$ & $1.2 \mathrm{e}-2$ \\
stiff & $7.8 \mathrm{e}-3$ & $5.9 \mathrm{e}-3$ \\
max stiff & $2.8 \mathrm{e}-3$ & $2.5 \mathrm{e}-3$ \\
\hline
\end{tabular}

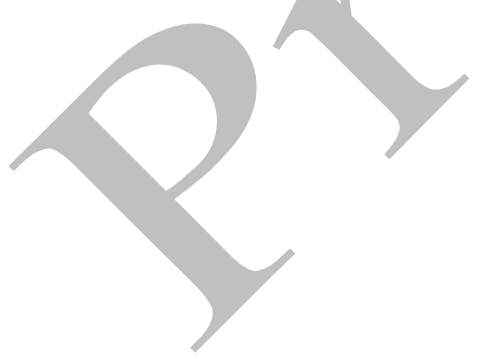




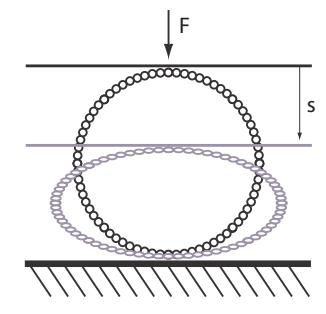

C

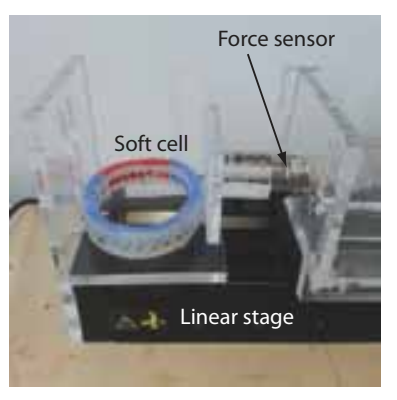

b

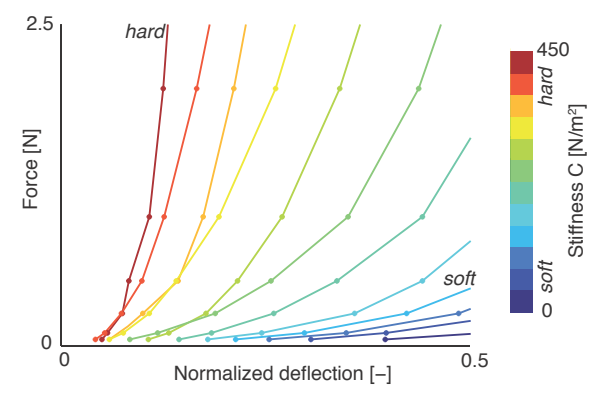

d

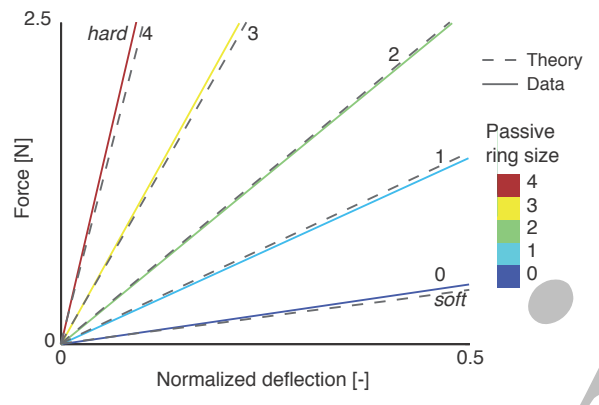

Figure S1: (a) Sketch of the force-deflection measurement setup in simulation, (b) data obtained in simulation for different internal pressure constant $\mathrm{C}$ settings and gradually increased force vs. deflection, (c) force-deflection measurement setup in hardware, (d) force vs. deflection data obtained for the fabricated cells when inserting different PDMS rings to change the cell softness.

a

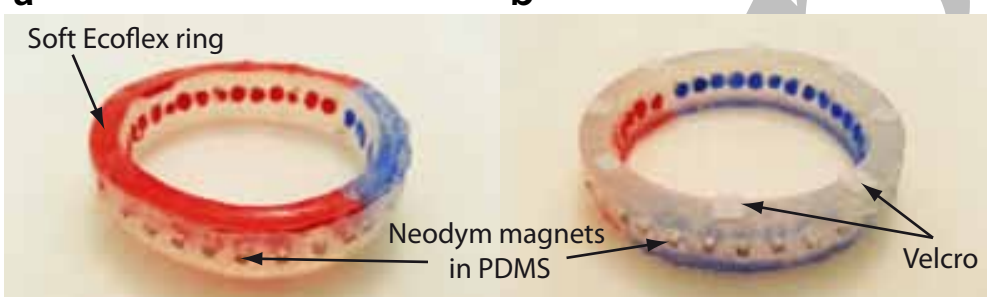

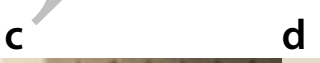

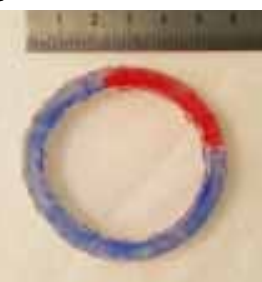

d

Stiffness rings

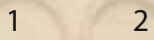

3
4

Figure S2: (a,b) Fabricated soft cells showing the Neodym magnets integrated in the cell membrane. (b) Small pieces of Velcro are glued underneath the cell to reduce ground friction. (c) Top-view of the cell measuring $5.5 \mathrm{~cm}$ in diameter. (d) Fabricated rings out of PDMS having different thickness that are inserted into the cells to change their softness.
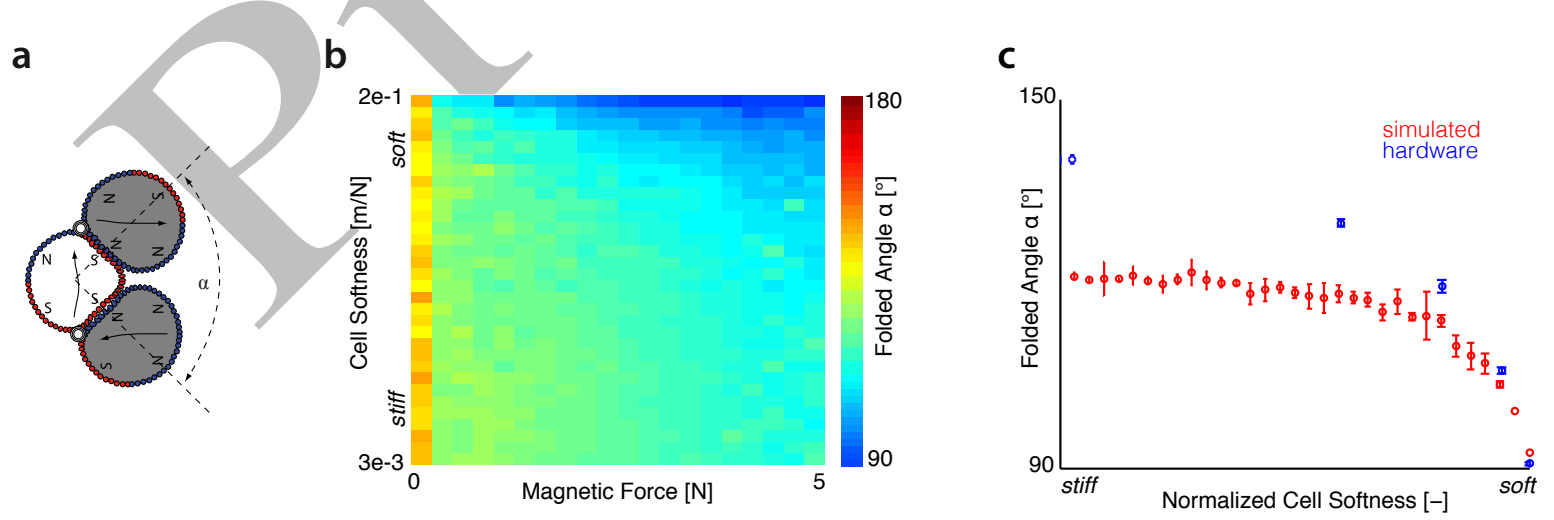

Figure S3: (a) Scheme of three consecutive cells in equilibrium illustrating the angle $\alpha$. (b) Angle $\alpha$ of three consecutive cells (color scale) as a function of cell softness and magnetic force in simulations. (c) Angle $\alpha$ of three consecutive cells as a function of normalized cell softness as measured with the hardware prototype (blue) and simulation (red). 